\title{
Treatment of Diffuse Diabetic Macular Oedema: A Comparison Between Argon and Krypton Lasers
}

\author{
A. G. CASSWELL, C. R. CANNING, Z. J. GREGOR \\ London
}

\begin{abstract}
Summary
We report the results of a randomised treatment trial of macular grid photocoagulation in 91 eyes with diffuse diabetic macular oedema followed up for at least two years comparing Krypton red with Argon blue/green lasers. At two years, macular oedema had improved equally in both groups, and the visual acuity was unchanged or better in $85 \%$ of eyes treated with Argon and in $79.5 \%$ of eyes treated with Krypton laser. No statistically significant differences were observed between the two groups in terms of visual acuity or the degree of oedema. We conclude that the observed effect of grid laser treatment in diffuse diabetic macular oedema did not depend on the wavelength of the laser light used in this study.
\end{abstract}

Macular oedema is present in about $9 \%$ of the diabetic population. ${ }^{1}$ It is more common in patients with maturity onset diabetes and it also occurs earlier in the course of the disease han in patients with juvenile onset diabetes. Focal oedema is characterised by focal fluorescein leakage from specific capillary lesions, and is often associated with rings of intraretinal exudate. Direct laser treatment of the leaking capillary lesions results in resorption of such rings. ${ }^{2}$

More widespread macular oedema and exudation may. result from multiple leaking lesions and is often associated with reduced visual acuity. In many cases, diffuse macular oedema is characterised by extensive retinal thickening with relatively few exudates, and widespread leakage not only from the retinal capillary bed but also from the underlying RPE. There may be associated areas of capillary non-perfusion and cystoid changes at the macula. The remaining capillaries are often dilated. Systemic factors, such as poor diabetic control, uncontrolled hypertension and renal failure have been associated with this form of macular oedema. ${ }^{3,4}$

Several clinical trials have demonstrated that grid photocoagulation for diffuse diabetic macular oedema is an effective method of reducing retinal oedema and can slow down or even reverse, the gradual decline in visual acuity which would otherwise occur if no treatment were given. ${ }^{5-10}$ In these studies argon blue-green laser was used for grid treatment.

There are a number of theoretical reasons why using wavelengths longer than those of argon blue/green may be more beneficial for macular treatment. Firstly, short wavelengths are more likely to be absorbed by the macular luteal pigment than longer wavelengths ${ }^{11}$ and this may induce unwanted macular toxicity. Secondly, destruction of inner retinal layers is common with shorter wavelength. ${ }^{12}$ Thirdly, there is experimental evidence in animals that retinal photocoagulation with long wavelengths, may have widespread beneficial effects on retinal capillary endothelium. ${ }^{13}$

A G Casswell and C R Canning were supported by The Friends of Moorfields.

Correspondence to: Z J Gregor, Moorfields Eye Hospital, City Road, London EC1V 2PD. 
The aim of the trial was to study the effects of grid laser treatment of patients with diffuse diabetic macular oedema comparing the short wavelength of argon blue/green with the longer wavelength of krypton red.

\section{Patients and Methods}

All patients were diabetic and were being treated with insulin or oral hypoglycaemic agents. They were included in this study if they had clinically significant macular oedema as defined by the Early Treatment Diabetic Retinopathy Study Research Group. ${ }^{14}$ The presence of one or more areas of retinal thickening, one or more disc diameters across, any part of which encroached within one disc diameter of the centre of the macula. Many had widespread retinal oedema and on fluorescein angiography, diffuse leakage was present.

Patients presenting to Moorfields Eye Hospital over a two year period were considered for inclusion into the study. The systemic exclusion criteria were uncontrolled hypertension, poorly controlled diabetes, and clinical evidence of cardiac or renal dysfunction with fluid overload. The ocular exclusion criteria were visual acuity worse than $6 / 60$, significant opacities in the ocular media such as cataract or vitreous haemorrhage, glaucoma, rubeosis, areas of capillary non-perfusion disrupting the perifoveal capillary arcade, proliferative retinopathy and previous laser treatment. Those patients unable to attend the hospital regularly or unable to comprehend the informed consent were excluded.

Patients were randomised by coin toss to receive grid photocoagulation with argon blue/green $(488 / 514 \mathrm{~nm})$ or krypton red $(647 \mathrm{~nm})$. Those patients eligible for treatment to both eyes received argon to one eye and krypton to the other.

Before treatment a full ophthalmic examination included slit lamp biomicroscopy, Goldman tonometry, stereo fundus photography and fluorescein angiography. The best corrected visual acuity was obtained by a member of the team who was not involved in the treatment, using the same room and the same Snellen chart on each occasion. Retinal thickness was assessed clinically using a +90 dioptre lens and/or a Goldman contact lens, as well as stereo photographs and was graded as no oedema, mild or severe oedema. The site and extent of fluorescein leakage and capillary dropout were noted on angiography.

Grid treatment was applied to the whole area of retinal thickening excluding the central 500 microns at the fovea. Spots of 200 microns were spaced one spot width apart using a duration of 0.1 second with sufficient power to produce a burn just visible immediately after treatment. No attempt was made to treat micro-aneurysms and the leaking capillary segments by direct photocoagulation. Treatment was not applied specifically to areas of ischaemia unless there was associated oedema.

Eyes were reassessed after three months, six months, and at six month intervals thereafter. Clinical examination and colour fundus photography were carried out on each occasion. Fluorescein angiography was repeated whenever further treatment was planned and/or when unexplained visual loss had occurred. Areas of residual oedema were retreated at these intervals using the same wavelength laser as was used originally, unless there were already clear photocoagulation scars at the site of the oedema.

Patients who developed significant cataracts had extracapsular extractions with or without lens implantation and remained in the study. Eyes which developed new vessels on the disc or elsewhere received panretinal photocoagulation using the same wavelength laser as was used to treat the macular oedema.

\section{Results}

One hundred and forty two eyes were enrolled in the trial, 91 of whom have been followed for two or more years. The age range was 30 to 90 years, with a mean of 62 (for Argon: 63 years, for Krypton: 61 years). There were 48 eyes treated with Argon (22 males, 26 females) and 43 eyes with Krypton (20 males and 23 females). Twenty-seven patients had both eyes treated and 37 had one eye treated. Controlled systemic hypertension was present in eight patients, and renal dysfunction without fluid overload in four. Multiple treatments were required with both Argon and Krypton (Table I). Each eye was treated on average every third visit.

The visual acuity change is detailed in Table 
Table I Details of Treatment

\begin{tabular}{|c|c|c|}
\hline $\begin{array}{l}\text { Number of treatments } \\
\text { per eye }\end{array}$ & $\begin{array}{l}\text { Argon } \\
\text { (48 eyes) }\end{array}$ & $\begin{array}{l}\text { Krypton } \\
\text { (43 eyes) }\end{array}$ \\
\hline 1 & 14 & 12 \\
\hline 2 & 21 & 18 \\
\hline 3 & 8 & 9 \\
\hline 4 & 3 & 4 \\
\hline More than 4 & 2 & 0 \\
\hline \multicolumn{3}{|l|}{$\begin{array}{l}\text { Number of burns per } \\
\text { treatment }\end{array}$} \\
\hline$<50$ burns & 35 & 24 \\
\hline $50-100$ burns & 34 & 37 \\
\hline$>100$ burns & 33 & 30 \\
\hline
\end{tabular}

II. After two years 6/48 (12.5\%), Argon treated eyes and 1/43 (2\%) Krypton treated eyes had improved by two or more lines of Snellen acuity. The majority (35/48 (73\%) Argon and 33/43 (77\%) Krypton) remained within one line of their pre-treatment acuity. Loss of vision by two or more lines of Snellen occurred in $7 / 48(15 \%)$ of eyes treated with Argon and 9/43 (21\%) of those treated with Krypton.

The effect of treatment on the degree of macular oedema is detailed in Table III. Overall patients in the Argon group had more improvement after two years than those in the Krypton group, but the differences were not statistically significant. No short-term deterioration in oedema was noted at the three month post-treatment visit.

Cataracts which progressed during the study required surgery in six Argon and four Krypton treated eyes. Proliferative retinopathy occurred and was treated with pan-retinal photocoagulation in one eye in the Argon group and five eyes in the Krypton group. One of the eyes in the Krypton group developed rubeosis iridis. One eye in the Argon

Table II Visual acuity at two years

\begin{tabular}{lcccc}
\hline & $\begin{array}{c}\text { Change in Visual Acuity at Two Years } \\
(>2 \text { lines of Snellen })\end{array}$ \\
\hline $\begin{array}{l}\text { Pre-treatment } \\
\text { visual acuity }\end{array}$ & total & better & same & worse \\
\hline $6 / 5$ to $6 / 9$ & A 14 & 0 & 12 & 2 \\
& K 18 & 0 & 15 & 3 \\
$6 / 12$ to 6/18 & A 22 & 3 & 17 & 2 \\
$6 / 18$ to 6/36 & K 11 & 0 & 8 & 3 \\
& A 12 & 3 & 6 & 3 \\
& K 14 & 1 & 10 & 3 \\
\hline
\end{tabular}

group developed subretinal neovascularisation at the site of a grid treatment burn.

\section{Discussion}

The results of this study support previously published reports of the value of grid laser photocoagulation in diffuse diabetic macular oedema, in as much as the treatment reduced intra-retinal leakage and helped to maintain the pre-treatment visual acuity. $5,6,8,9,10$

In diffuse macular oedema, there is widespread retinal vascular leakage (breakdown of inner blood-retinal barrier), but careful examination of stereo pairs of fluorescein angiograms often demonstrates a relatively early appearance of dye in the deeper retinal layers and in the pigment epithelium (breakdown of the outer blood-retinal barrier). In a large proportion of our patients such 'deep' leakage predominated and thus justified directing the treatment at the site of the outer blood-retinal barrier, the pigment epithelium. The lack of difference between the outcome of the patients treated with short wavelength (Argon blue/green) and those treated with long wavelengths (Krypton red), was perhaps disappointing, although not altogether surprising.

The underlying principles of grid laser photocoagulation are poorly understood, but there are a number of theoretical reasons why longer wavelengths should be as effective, or indeed more so than the shorter wavelengths. Although initially the destruction of focal areas of pigment epithelium may lead temporarily to worsening of the oedema, ${ }^{9}$ the pigment epithelium regenerates by mitosis and sliding. ${ }^{15}$ It may be that following such 'debridement' of the sick pigment epithelium, new, more vigorous cells take over the task of pumping out the excess retinal fluid as well as establishing better tight cell junctions ('rejuvenated' outer blood-retinal barrier). Both Krypton red and Argon blue/green are well

Table III Macular Oedema at Two Years

\begin{tabular}{lcccc}
\hline $\begin{array}{l}\text { Pre-treatment } \\
\text { macular oedema }\end{array}$ & total & none & mild & severe \\
\hline Mild & A 18 & 16 & 2 & 0 \\
& K 16 & 9 & 6 & 1 \\
Severe & A 30 & 11 & 11 & 8 \\
& K 27 & 12 & 7 & 8 \\
\hline
\end{tabular}


absorbed by the pigment epithelium. In addition, Krypton red is absorbed by the melanocytes of the choroid. Although there is evidence that there are choroidal changes in patients with diabetic retinopathy, ${ }^{16,17}$ it is not known whether selective photocoagulation of the choroid influences the course of diabetic maculopathy.

The destruction of the outer retinal layers is thought to reduce the metabolic demand of the outer retina and to improve the oxygenation of the inner retina. ${ }^{3}$ It has been shown histologically ${ }^{12}$ that photocoagulation with Krypton, but not with Argon laser, causes selective destruction of the outer retinal layers with minimal or no disturbance of the inner retinal layers. One would therefore expect a greater beneficial effect in the Krypton treated group; however, there was no evidence of such difference between the two groups on clinical examination, or in terms of the visual acuities.

In this study, it could be argued that the absorption of shorter wavelengths by blood in retinal capillaries may have resulted in such an unintentional direct (focal) coagulation of the leaking retinal vessels. However, the diameter of such leaking vessels is usually smaller than the cross-section diameter of the irradiating beam used in this study $(200 \mu)$ and thus the energy absorbed by the blood column within the vessel would be insufficient to produce thermal damage. We attempted to produce a minimal visible photocoagulation reaction, but the variable thickness of the oedematous retina at times caused a greater energy uptake and occasionally a full-thickness retinal burn resulted. Such burns would have involved the retinal capillaries, but it is unlikely that the slight trend in favour of the Argon treated group could be attributed to this effect alone.

The previously suggested effect of Krypton red on aretinal capillary proliferation in an animal model, ${ }^{13}$ was not obvious clinically. Marshall et al observed that localised grid laser photocoagulation induced retinal capillary proliferation in pig retina. This proliferation appeared to spread in waves throughout the retinal venous system well distal to the photocoagulation site. Krypton red laser was strikingly more effective in producing this capillary change than was Argon blue/green. The proposed explanation was that a mitogenic factor may be released by the damaged retinal pigment epithelium or is allowed to permeate into the retina. If such endothelial proliferation occurs in man, then grid photocoagulation would be beneficial for the restoration of the inner blood retinal barrier throughout the capillary bed of the posterior pole. The hope was that this would in turn improve the capillary integrity and reduce the efflux of fluid into the neuro-retina. The results of our study however, do not validate this hypothesis. Although reduced leakage from the capillary bed occurred in most patients, there was no observable difference between the Argon and Krypton treated groups at two years. It is possible that with longer follow up a difference between the two groups will become apparent. Meanwhile, further studies both in animals and in humans are needed to investigate this difficult area of management of diabetic retinopathy.

\section{References}

${ }^{1}$ Klein R, Klein BEK, Moss SE, et al.: The Wisconsin epidemiologic study of diabetic retinopathy. IV Diabetic macular edema. Ophthalmology 1984, 91: 1464-74.

${ }^{2}$ Spalter HF: Photocoagulation of circinate maculopathy in diabetic maculopathy. Am J Ophthalmol 1971, 71: 242-50.

${ }^{3}$ Bresnick GH: Diabetic maculopathy-a critical review highlighting diffuse macular edema. $O p h$ thalmology 1983, 90: 1301-17.

${ }^{4}$ Bresnick GH, Engerman R, Davis MD, et al.: Patterns of ischaemia in diabetic retinopathy. Trans Am Acad Ophthalmol Otolaryngol 1976, 81: 694-709.

${ }^{5}$ Patz A, Schatz H, Berkow JW, et al.: Macular oedema-an overlooked complication of diabetic retinopathy. Trans Am Acad Ophthalmol Otolaryngol 1973, 77: 34-42.

${ }^{6}$ Blankenship GW: Diabetic macular edema and argon laser photocoagulation for diffuse diabetic macular edema. Ophthalmology 1979, 86: 69-78.

${ }^{7}$ British Multicentre Study Group. Photocoagulation for diabetic maculopathy. Diabetes 1983, 32: 1010-16.

${ }^{8}$ Early treatment diabetic retinopathy study research group. Photocoagulation for diabetic macular edema. ETDRS Report No. 1. Arch Ophthalmol 1985, 103: 1796-806.

${ }^{9}$ Olk JR: Modified grid argon (blue/green) laser photocoagulation for diffuse diabetic macular edema. Ophthalmology 1986, 93: 938-50.

${ }^{10}$ Whitelock RAE, Kearns M, Blach RK, Hamilton AM: The diabetic maculopathies. Trans Ophthalmol Soc UK 1979, 99: 314-18. 
${ }^{11}$ Geeraets WJ \& Berry ER: Ocular spectral characteristics as related to hazards from lasers and other light sources. Am J Ophthalmol 1968, 66: 15-30.

${ }^{12}$ Marshall J \& Bird AC: A comparative histopathological study of argon and krypton laser irradiations of the human retina. BrJ Ophthalmol 1979, 63: $657-68$.

${ }^{13}$ Marshall J, Clover G, Rothery S: Some new findings on retinal irradiation by krypton and argon lasers. Doc Ophthalmol Proc Ser 1984, 36: 21-37.

${ }^{14}$ Early treatment diabetic retinopathy study research group. Treatment techniques and clinical guidelines for photocoagulation of diabetic macular edema. ETDRS Report No. 2. Ophthalmology 1987, 94: 761-74.

${ }^{15}$ Wallow IH: Repair of the pigment epithelium barrier following photocoagulation. Arch Ophthalmol 1984, 102: 126-35.

${ }^{16}$ Hydayait AA and Fine BS: Diabetic choroidopathy. Light and electron microscopic observations of seven cases. Ophthalmology 1985, 92: 512-22.

${ }^{17}$ Yanoff M: Ocular pathology of diabetes mellitus. Am J Ophthalmol 1969, 67: 21-38. 